\title{
A new path to treating arthritis?
}

Vascular permeability is a major drawback of anticytokine therapy for inflammatory diseases. However, researchers from the University of Utah have now shown that it might be possible to treat inflammation while avoiding endothelial dysfunction.

Investigations by Dean Li and colleagues, now published in Nature, demonstrate that a novel IL-1 $\beta$-activated intracellular signalling pathway is responsible for the cytokine's effects on endothelial cell-cell interaction, independent of canonical NFkB-mediated transcription and its targets. Disruption of MyD88-IRAK1 signalling in human dermal vascular endothelial (HMVEC-d) cells blocked nuclear translocation of NFkB but did not interfere with vascular permeability or cellsurface localization of vascular endothelial cadherin (VE-cadherin, an adhesion molecule important to the maintenance of endothelial integrity).

Subsequent investigations pointed to MyD88-dependent ADP-ribosylation factor 6 (ARF6) and its activator ARF nucleotide-binding site opener (ARNO; also known as cytohesin-2) as critical regulators of vessel destabilization, through effects on localization of VE-cadherin to the cell surface. Consistent with this idea, treatment of HMVEC-d cells with the cytohesin

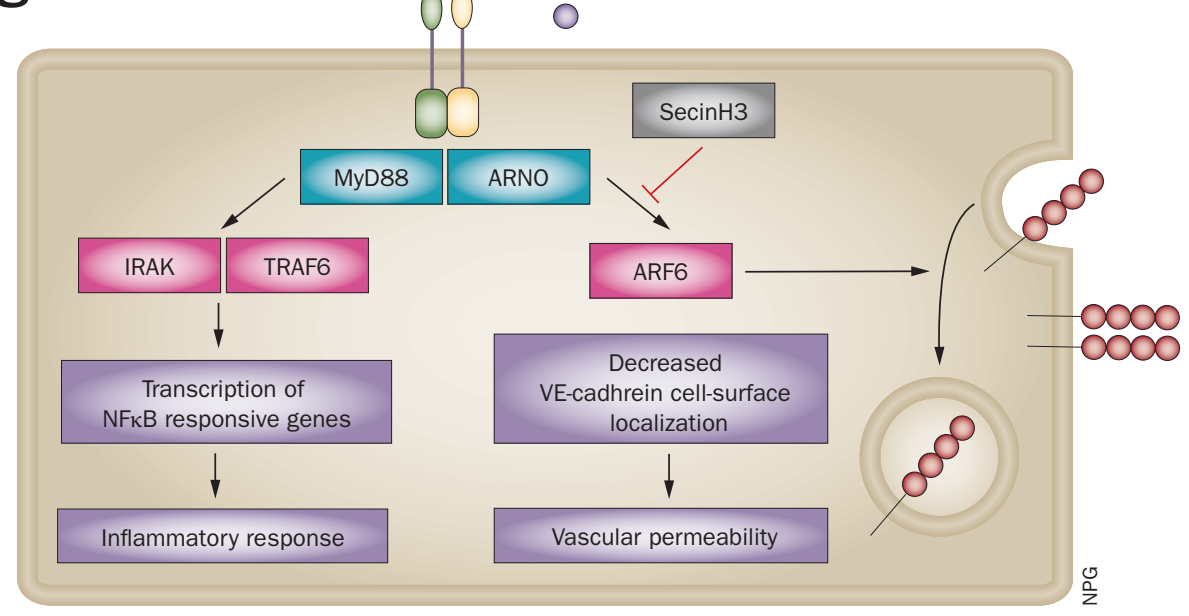

inhibitor SecinH3 disrupted IL-1 $\beta$ induced cell-surface localization of VE-cadherin and endothelial permeability. Notably, SecinH3 did not disrupt the NFkB pathway, suggesting that this pathway and the one mediated by ARNO-ARF6 diverge at MyD88.

In the collagen-induced animal model of arthritis, SecinH3 inhibited articular inflammation, pannus formation, cartilage damage and bone damage to a similar extent as the anti-TNF agent etanercept. The ARNO inhibitor decreased vascular permeability in arthritis joints, but did not affect cytokine levels $24 \mathrm{~h}$ after treatment.

The researchers contend that inhibition of the MyD88-ARNO-ARF6 pathway could exclusively target vascular permeability without interfering with important NFkB-dependent functions. "We believe that this noncanonical cytokine signalling pathway opens the possibility of limiting the destructive effects of cytokines on normal tissues without causing massive immunosuppression as occurs with current anticytokine therapies," says Li.

Sarah Onuora

Original article Zhu, W. et al. Interleukin receptor activates a MYD88-ARNO-ARF6 cascade to disrupt vascular stability. Nature doi:10.1038/nature11603 